\title{
Synapses and Sisyphus: life without paraplegin
}

\author{
Harris A. Gelbard \\ Departments of Neurology, Pediatrics, and Microbiology and Immunology; \\ and the Center for Aging and Developmental Biology; \\ University of Rochester Medical Center, Rochester, New York, USA
}

The family of neurodegenerative diseases known as hereditary spastic parapareses have diverse genetic loci, yet there is a remarkable convergence in the neuropathologic and neurologic phenotype. A report describing the construction of a transgenic mouse with a deletion of a nuclear-encoded mitochondrial protein involved in the regulation of oxidative phosphorylation suggests that this family of diseases may reflect activation of a final common pathway involving synaptic dysfunction that progresses to destruction of the presynaptic nerve terminal and axon (see the related article beginning on page 231).

J. Clin. Invest. 113:185-187 (2004). doi:10.1172/JCI200420783.

Hereditary spastic paraplegia (HSP) is an umbrella term for a group of neurodegenerative diseases with diverse clinical presentations and patterns of inheritance including autosomal dominant, autosomal recessive, and $\mathrm{X}$ linked. Indeed, 19 loci for HSP have been identified thus far (1). The unifying clinical symptom is progressive, often severe, symmetric lower-extremity spasticity that, in isolation, is known as pure HSP (PHSP) and, when accompanied by other deficits including impaired position sense and bladder disturbance, is known as complicated HSP (CHSP). Despite the genetic diversity inherent in this group of disorders, considerable progress has been made in defining pathogenetic mechanisms for at least some forms of HSP.

\section{Paraplegin and HSP}

In this issue of the JCI, Ferreirinha et al. (2) describe the design and characterization of a transgenic mouse with

\footnotetext{
Address correspondence to: Harris A. Gelbard, Center for Aging and Developmental Biology, Aab Biomedical Institute, University of Rochester Medical Center, Box 645, 601 Elmwood Avenue, Rochester, New York 14642, USA. Phone: (585) 273-1473; Fax: (585) 506-1947; E-mail: Harris_Gelbard@urmc.rochester.edu. Conflict of interest: The author has declared that no conflict of interest exists.

Nonstandard abbreviations used: hereditary spastic paraplegia (HSP); pure HSP (PHSP); complicated HSP (CHSP).
}

a targeted deletion of $\operatorname{Spg} 7\left(\operatorname{Spg} 7^{-/}\right)$ that is associated with a clinical phenotype strikingly similar to HSP. A report by Settasatian et al. (3) characterized the $S p g 7$ gene as a nuclearencoded mitochondrial metalloprotease that maps to chromosome $16 \mathrm{q} 24.3$ and is the genetic locus for an autosomal recessive form of HSP. This metalloprotease, also known as paraplegin, shares a high degree of homology with yeast mitochondrial ATPdependent zinc metalloproteases, including AFG3, RCA1, and YME1, which have proteolytic and chaperonelike activities at the inner mitochondrial membrane $(4,5)$. A report by Casari et al. (6) found a deletion mutation in paraplegin from familial kindred with a 16q24.3 linkage, as well as several frameshift mutations in individuals with CHSP and PHSP. Muscle biopsies from two of these patients demonstrated defects in oxidative phosphorylation, despite the fact that paraplegin is not a protein associated with oxidative-phosphorylation abnormalities per se (7). Most recently, the work of Atorino et al. (8) suggests a possible mechanism for this observation. These investigators demonstrate that paraplegin coassembles with a homologous protein, AFG3L2, also present in the inner mitochondrial membrane, into a high-molecular mass complex. Using fibroblasts from patients with HSP, they showed that this complex is defective, with a resultant decrease in mitochondrial complex I activity and increased sensitivity to oxidant stress. Exogenous expression of wild-type paraplegin ameliorated both of these deficits. Finally, these investigators used yeast-complementation studies to demonstrate that the parapleginAFG3L2 complex is functionally conserved with the yeast matrix-ATPaseassociated activities protease, suggesting that this complex possesses proteolytic activity.

The neuropathology of Spg $7^{-/-}$mice The Spg $7^{-/-}$mice created by Ferreirinha et al. (2) develop a neuropathologic phenotype of a distal axonopathy characterized by swelling and degeneration in spinal and peripheral axons. Although the axonopathy does not occur until 8 months of age, Spg $7^{-1-}$ mice develop mitochondrial abnormalities in their axons at 4.5 months of age, a time when they already exhibit motor deficits as evidenced by impaired ability to maintain their balance on a rotating rod. These mitochondrial abnormalities include hypertrophy, concentric cristae, herniations, and the appearance of giant mitochondria. Mitochondria with concentric cristae are often seen in normal and pathologic conditions with increased cellular metabolic activity.

Two additional striking findings emerge from this study: at every age studied, the percentage of axons containing abnormal mitochondria greatly exceeded the number of swollen or degenerated axons; and abnormal mitochondria were present in synaptic terminals in the anterior horn of the lumbar spinal cord at 4.5 months of age (2). Thus, abnormal mitochondria in nerve terminals are the earliest neuropathologic hallmark of Spg $7^{-/-}$mice. A diminution in ATP synthesis, however, is only apparent relatively late in the course of the disease (23-26 months of age). Interestingly, the other functional deficit studied in this report, retrograde transport in lumbar motor neurons, is not impaired in $S p g 7^{-/-}$mice until 17 months of age.

Thus, availability of the Spg $7^{-/-}$ model will allow investigators in the 


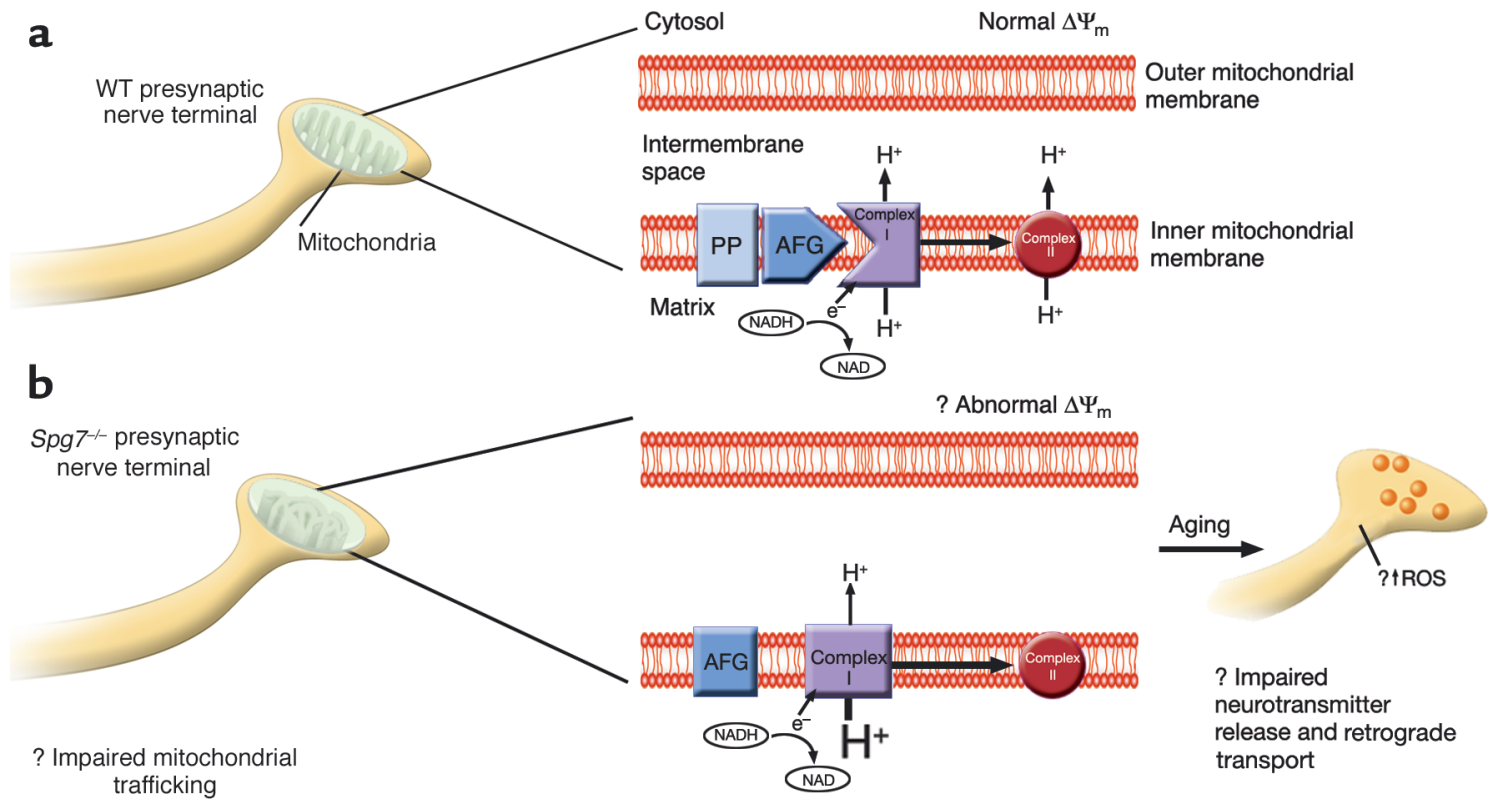

\section{Figure 1}

Hypothetical scheme for diminished complex I activity in presynaptic nerve terminals of Spg $7^{-1-}$ mice. (a) Schematic of a mitochondrion with a normal morphologic phenotype is depicted in a presynaptic nerve terminal, with an enlarged schematic of mitochondrial structure. Within the inner mitochondrial membrane, the nuclear-encoded product of Spg7, paraplegin (PP), forms an approximately 900-kDa complex with AFG3L2 (AFG) (7). In this hypothetical scheme, this complex has proteolytic activity that is necessary for the normal physiologic activity of $\mathrm{NADH}$-ubiquinone oxidoreductase (complex I), such that the normal proton gradient is maintained between the mitochondrial matrix and the intermembrane space. This in turn allows for normal functioning of oxidative phosphorylation, and preservation of the mitochondrial membrane potential $\left(\Delta \psi_{\mathrm{m}}\right)$. However, in the absence of paraplegin in nerve terminals from an Spg $7^{-1-}$ mouse (b), an abnormal complex of AFG3L2 with a molecular weight of about $250 \mathrm{kDa}(7)$ forms in the inner mitochondrial membrane that is unable to induce the necessary sequence of proteolytic events such that complex I has normal activity. This in turn results in a decrease in the proton gradient between the matrix and intermembrane space, with a presumed change in $\Delta \psi_{\mathrm{m}}$. As the animal ages, the net effect of diminished complex I activity in presynaptic nerve terminals is likely to be impairment of neurotransmitter release, pathologic generation of reactive oxygen species (ROS), and decreased retrograde transport of trophic substances.

field to extend the observations of Atorino et al. (8) regarding loss of the complex formed by paraplegin and the murine homolog of AFG3L2 in the inner mitochondrial membrane, and whether this loss results in similar decreases in complex I activity and susceptibility to oxidant stress. This is especially intriguing in view of the observation that complex I is preferentially affected by age-related changes in oxidative stress (9), with the most significant dysfunction observed in synaptic mitochondria (10). In particular, synaptic mitochondria show a differential sensitivity to inhibitors of complex I, III, and IV activity, such that complex I is threefold more sensitive to inhibition with respect to oxygen consumption and ATP production.

In light of these findings, the demonstration that the morphology of synaptic mitochondria are preferentially affected in $S p g 7^{-/-}$mice as the earliest neuropathologic hallmark of this transgene, suggests a possible mechanism for the neurologic deficits in these animals (Figure 1). As the animal ages, synaptic activity becomes progressively more compromised with increasing metabolic demands because of impaired complex I activity and increasing oxidative stress. This ultimately results in a dying-back axonopathy, with dissolution of the nerve terminal occurring first, followed by destruction of the more proximal parts of the axon. Because motor deficits are co-temporaneous with the appearance of abnormal mitochondria in synaptic terminals, a parallel can be drawn with the fate of the Greek king Sisyphus, condemned forever to roll a boulder up a hill, only to have it roll back down on him. Specifically, the formation of synapses is a normal part of development in the nervous system, but in HSP, synapse forma- tion and normal function in affected pathways are doomed to failure in a retrograde fashion. The finding that ATP production is decreased quite late (23-26 months) in the course of the animal's lifespan does not necessarily confound this scheme, since there may be other compensatory mechanisms at play to conserve energy production in these animals. Regardless, there is a considerable temporal window of opportunity to rescue affected mitochondria in Spg $7^{-1-}$ mice by introduction of wildtype paraplegin prior to the onset of motor symptoms and the appearance of abnormal mitochondria. This type of study will afford investigators significant insights into whether there is a critical period for amelioration of the clinical phenotype of HSP. Despite the relative diversity of genetic loci for HSP, there is a convergent neuropathologic phenotype. Ultimately the phenomenon of 
synaptic dysfunction with retrograde destruction of axons may occur because a final common pathway is activated for presynaptic neurodegeneration, just as excitotoxicity represents a molecular metaphor for many postsynaptic forms of neurodegeneration. Thus, data from experiments investigating mitochondrial and synaptic function in Spg $7^{-/-}$ mice will likely yield valuable insights into new therapeutic avenues targeted at rescue of energy metabolism and modulation of oxidative stress in the synapse.

\section{Acknowledgments}

The author is supported by grants from the NIH (P01 MH64570, R01 MH56838, and P01 NS31492).

1. Reid, E. 2003. Many pathways lead to hereditary spastic paraplegia. Lancet Neurol. 2:210.

2. Ferreirinha, F., et al. 2004. Axonal degeneration in paraplegin-deficient mice is associated with abnormal mitochondria and impairment of axonal transport. J. Clin. Invest. 113:231-242. doi:10.1172/JCI200420138.

3. Settasatian, C., et al. 1999. Genomic structure and expression analysis of the spastic paraplegia gene, SPG7. Hum. Genet. 105:139-144.

4. Coppola, M., et al. 2000. Identification and characterization of YME1L1, a novel paraplegin-related gene. Genomics. 66:48-54.

5. Kremmidiotis, G., et al. 2001. Molecular and functional analyses of the human and mouse genes encoding AFG3L1, a mitochondrial metalloprotease homologous to the human spastic paraplegia protein. Genomics. 76:58-65.

6. Casari, G., et al. 1998. Spastic paraplegia and OXPHOS impairment caused by mutations in paraplegin, a nuclear-encoded mitochondrial metalloprotease. Cell. 93:973-983.

7. Orth, M., and Schapira, A.H. 2001. Mitochondria and degenerative disorders. Am. J. Med. Genet. 106:27-36.

8. Atorino, L., et al. 2003. Loss of m-AAA protease in mitochondria causes complex I deficiency and increased sensitivity to oxidative stress in hereditary spastic paraplegia. J. Cell Biol. 163:777-787. 9. Wong, A., et al. 2002. Differentiation-specific effects of LHON mutations introduced into neuronal NT2 cells. Hum. Mol. Genet. 11:431-438.

10. Davey, G.P., Peuchen, S., and Clark, J.B. 1998 Energy thresholds in brain mitochondria. Potential involvement in neurodegeneration. J. Biol. Chem. 273:12753-12757. 